\title{
Bacillus cereus
}

National Cancer Institute

\section{Source}

National Cancer Institute. Bacillus cereus. NCI Thesaurus. Code C86161.

A species of aerobic or facultatively anaerobic, Gram-positive, rod shaped bacteria assigned to the phylum Firmicutes. This species is motile, spore forming, and catalase positive, hydrolyzes casein, starch, and gelatin, and produces the toxin cereulide. B. cereus is found in soil and associated with diarrheal and emetic forms of food-borne illness as well as opportunistic infections in both humans and domestic animals. 